\title{
Cyclic oscillations of leucocyte counts in chronic myeloid leukaemia
}

\author{
O.P. Malhotra ${ }^{1}$ and S. Roy Salam ${ }^{2}$
}

Departments of ${ }^{1}$ Medicine and ${ }^{2}$ Laboratory Medicine, All India Institute of Medical Sciences, Ansari Nagar, New Delhi-110029, India

Summary: A patient with chronic myeloid leukaemia, having two unusual features, is described. He had absence of splenomegaly and marked periodic fluctuations of leucocyte counts, occurring spontaneously, in the initial phase of the disease. The latter finding suggests that, at least in some patients with chronic myeloid leukaemia, negative feed back control mechanism(s), possibly exerted by colony stimulating factor, may be retained which may have therapeutic implications.

\section{Introduction}

Chronic myeloid leukaemia (CML), the commonest type of leukaemia in India, usually presents with considerable splenomegaly, high leucocyte count, low leucocyte alkaline phosphatase (LAP) score and the presence of Philadelphia $(\mathrm{Ph}+)$ chromosomal abnormality. Absence of splenomegaly occurs in less than $5 \%$ of patients. ${ }^{1}$ Similarly there are very few reports of spontaneous cyclic oscillations of the leucocyte counts in these patients..$^{2-7}$ In this communication, we present a patient who had these two unusual features during the initial phase of his illness.

\section{Case report}

A 54 year old male presented to us in September 1988 with a history of pain and swelling in the left leg and large red and blue ecchymotic patches over the shoulder, hips and thighs of 16 days duration. He was known to have uncomplicated ischaemic heart disease and non-insulin dependent diabetes mellitus.

Physical examination revealed a few ecchymotic patches over the extremities and evidence of deep vein thrombosis in the left leg. The rest of the physical examination was unremarkable. There was no splenomegaly or bone tenderness.

Haematological profile revealed a haemoglobin level of $11.5 \mathrm{~g} / \mathrm{dl}$, total leucocyte count $148 \times 10^{9} / 1$ with neutrophils $60 \%$, stab forms $15 \%$, metamyelocytes $10 \%$, myelocytes $5 \%$, monocytes $5 \%$ and lymphocytes $5 \%$. In view of these findings, his age, absence of splenomegaly, and presence of deep vein

Correspondence: O.P. Malhotra, M.D.

Accepted: 24 July 1990 thrombosis, he was considered to have either leukaemoid reaction secondary to a possible underlying malignancy or CML. The latter diagnosis was subsequently confirmed on the basis of low LAP score (LAP score of patient $=2$, control $=60$ ) and the presence of $\mathrm{Ph}+$ chromosome.

He was followed up with or without therapy and subsequent haematological findings, reflected in Figure 1, showed the interesting phenomenon of spontaneous oscillations of leucocyte counts. He maintained high leucocyte counts for a period of 50 to 60 days interrupted by a period of normal leucocyte counts for a period of 20 to 30 days. Haemoglobin levels also showed similar fluctuations (Figure 1). Platelets were not counted but were found to be adequate on the basis of peripheral blood film examination. It is evident that treatment with busulphan either alone or with hydroxyurea did not modify the cyclic pattern of the leucocyte counts or the haemoglobin levels.

In July 1989 there was a sudden transformation of the chronic phase of CML into acute lymphoblastic leukaemia. There was no intervening evidence of accelerated phase of the disease. At the time of blast crisis the total leucocyte count was $16.8 \times 10^{9} / 1$ with $40 \%$ blasts present in the peripheral smear. There was no splenomegaly. He had a temporary remission with vincristine and prednisolone combination therapy before he relapsed and died in November, 1989.

\section{Discussion}

The leucocyte count usually rises exponentially in patients of CML. However, some of these patients are reported to have periodic fluctuations of the leucocyte counts with or without therapy. ${ }^{2-7}$ The 


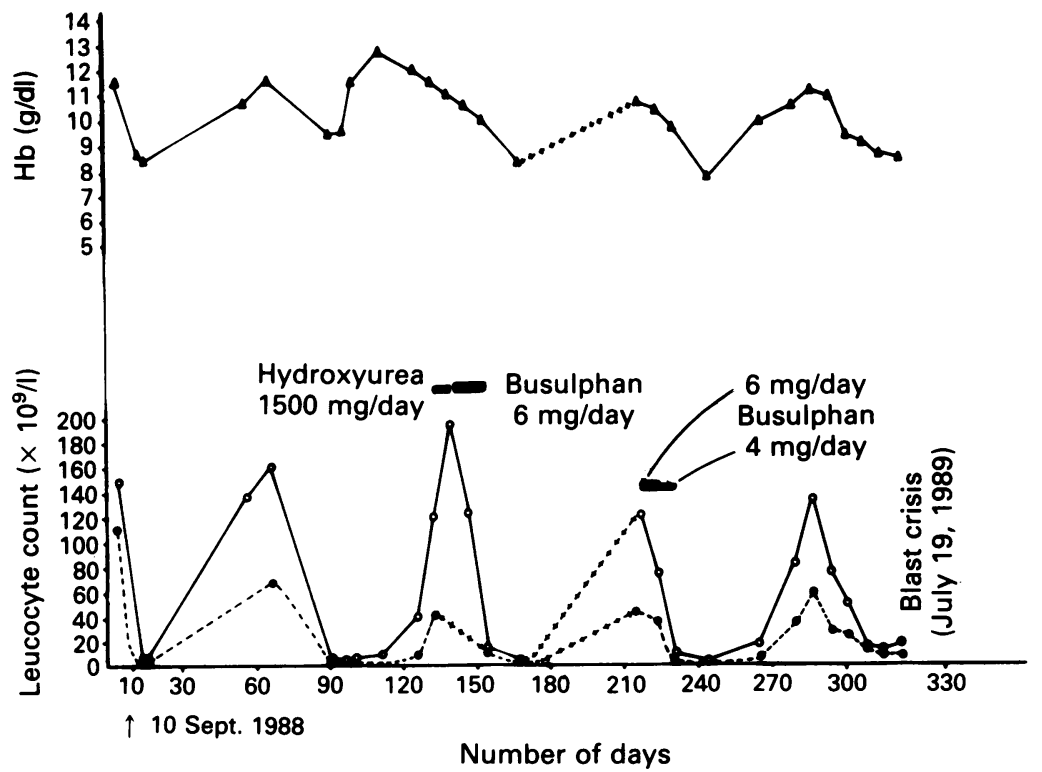

Figure 1 Showing cyclic oscillations of leucocyte counts and haemoglobin levels. O-O, Total leucocyte count; - - , absolute neutrophil count; $O_{--} \mathrm{O}$, no records available.

crest to crest interval has been observed to vary from 30 to 120 days while the amplitude of the leucocyte counts has been reported to range from as low as $0.99 \times 10^{9} / 1$ to as high as $269 \times 10^{9} / 1^{6,7}$ Our patient had an intercrest interval of 60 to 75 days and the amplitude of the leucocyte counts ranged from $3 \times 10^{9} / 1$ to $194 \times 10^{9} / 1$. A similar oscillation of leucocyte counts with shorter intervals and lower amplitude has long been observed in some healthy individuals. ${ }^{8}$ It has therefore been suggested that the granulocyte production by the bone marrow is cyclical, being controlled by a negative feed back circuit and is not due to any selective shifting between the circulating and the marginating leucocyte pools. ${ }^{4}$ The negative feed back control is believed to be exerted by colony stimulating factor. ${ }^{2,4,7,9}$ Periodic fluctuations in the reticulocyte and platelet counts have also been described..$^{2,4,7}$ Our patient also showed periodic variations of the haemoglobin levels in phase with the leucocyte counts. Furthermore similar patterns of cyclic oscillations of the leucocyte counts were maintained with or without therapy. These observations suggest that CML may not always be an autonomous neoplasm with unrestrained cellular growth. Rather, a feed back control mechanism, though partial, may possibly be retained in some of these patients.

Another interesting feature observed in our patient was the absence of splenomegaly at the onset of the disease. This is not a common finding, being usually reported in less than $5 \%$ of patients of CML. ${ }^{1}$ Besides the conventional treatment, other therapeutic options exist for such patients. It may be possible to control the high leucocyte count by means of leucapheresis or by suitably modifying the feed back mechanism(s). The findings in our patient reinforce the need to study the natural history and pathophysiology of the disease in greater detail particularly the feed back control mechanism(s). Such studies may lead to the development of suitable therapeutic interventions with the ultimate objective of curing this largely fatal condition.

\section{References}

1. Wintrobe, M.M. Chronic myeloid leukaemia. In: Wintrobe, M.M., Lee, G.R., Boggs, D.R. et al. (eds) Clinical Haematology, 8th edition. Lea and Febiger, Philadelphia, 1981, p. 1566.

2. Chikkappa, G., Borner, G., Burlington, H. et al. Periodic oscillation of blood leucocytes, platelets and reticulocytes in a patient with chronic myelocytic leukaemia. Blood 1976, 47: 1023-1030. 
3. Gatti, R.A., Robinson, W.A., Deinard, A.S. et al. Cyclic leucocytosis in chronic myelogenous leukaemia: new perspectives on pathogenesis and therapy. Blood 1973, 41: 771-782.

4. Vodopick, H., Rupp, E.M., Edwards, C.L., Goswitz, F.A. \& Beauchamp, J.J. Spontaneous cyclic leucocytosis and thrombocytosis in chronic granulocytic leukaemia. $N$ Engl $J$ Med 1972, 286: 284-290.

5. Kennedy, B.J. Cyclic leucocyte oscillations in chronic myelogenous leukaemia during hydroxyurea therapy. Blood 1970 , 35: $751-760$.

6. Morley, A.A., Baikie, A.G. \& Galton, D.A.G. Cyclic leucocytosis as evidence for retention of normal homeostatic control in chronic granulocytic leukaemia. Lancet 1967, ii: 1320-1323.
7. Rodriguez, A.R. \& Lutcher, C.L. Marked cyclic leucocytosis leucopenia in chronic myelogenous leukaemia. Am J Med 1976, 60: $1041-1047$.

8. Morley, A.A. A neutrophil cycle in healthy individuals. Lancet 1966, ii: $1220-1222$.

9. Robinson, W.A. \& Pike, B.I. Leucopoietic activity in human urine. The granulocytic leukaemias. $N$ Engl J Med 1970, 282: 1291-1297. 